\title{
Multicast Streaming over 3G Cellular Networks through Multi-Channel Transmissions: Proposals and Performance Evaluation
}

\author{
Michele Rossi ${ }^{\dagger}$, Paolo Casari ${ }^{\star}$, Marco Levorato ${ }^{\star}$, Michele Zorzi ${ }^{\star}$
}

\begin{abstract}
In this paper, we propose a novel technique for the provisioning of multicast streaming flows in 3G W-CDMA cellular systems. Our focus here is on the transmission of a downlink multicast streaming flow to the interested users in a $3 G$ cell. Over such networks, due to multi-user interference and channel impairments such as, e.g., deep fading phenomena, some error control features have to be provided in order to meet QoS guarantees. In previously proposed work [1], the wanted error resilience is achieved by exploiting common channel $(\mathrm{CCH})$ transmissions and retransmitting lost packets according to multi-users HARQ schemes. Here, we propose to substitute ARQ error recovery with packet-based Forward Error Correction (FEC) [2] and with the transmission of a certain amount of redundancy over paralle common channels. In practice, we exploit the temporal diversity over multiple channels to strengthen the FEC scheme thereby increasing the QoS. In this way, every user can independently join the desired number of channels in order to optimize its own quality and without affecting the performance of the other users in the system. To increase error resilience, in every channel we exploit well-known packet-based Reed Solomon like coding techniques [2]. Moreover, appropriate time shifts of the information sent across the parallel CCH channels are also introduced to increase the robustness against error bursts. The performance evaluation is carried out through an analytical framework. The obtained results show the substantial benefits deriving from the usage of a multiple channel transmission technique.
\end{abstract}

\section{INTRODUCTION}

$\mathbf{I}$ $\mathrm{N}$ the last few years, a considerable amount of investments was paid by operators all over the globe for $3 \mathrm{G}$ spectrum licenses. Now, 3G systems are becoming a reality and good services, possibly superior to the ones existing in $2 \mathrm{G}$ cellular networks, are to be provided to the final users in order to encourage them to join this new technology, thereby leading to its success and to a return of the aforementioned investments. Of course, one of such services consists of the provisioning of multicast video streaming flows. The delivery of high bandwidth video flows is, in fact, one of the new possibilities offered by the $3 \mathrm{G}$ technology that must be exploited to encourage new users to join it. Algorithms for the efficient delivery of such flows are the main concern of this paper.

The focus here is on the transmission of a multicast streaming flow to a set of interested users in a $3 \mathrm{G}$ cell. Our goal is to devise a scalable, simple and still effective algorithm for the provisioning of such flow, where each user can dynamically adapt its reception quality, but without affecting the other users in the system. In this work, we take inspiration from

${ }^{\dagger}$ Department of Engineering, University of Ferrara via Saragat $1-44100$ Ferrara, Italy. ${ }^{\star}$ Department of Information Engineering, University of Padova, via Gradenigo 6/B - 35131, Padova, Italy. the proposal in [3], whose aim is to improve a multicast transmission over wired networks, and we extend this work to the case of the transmission of a multicast flow in a $3 \mathrm{G}$ cellular network. Our main contributions here are to devise a new way of performing error correction in the multicast streaming case and to provide a simple analytical framework in order to test the performance of the proposed scheme.

Several adaptive algorithms to increase and control the error resilience of video streaming flows in wired networks have been proposed so far [3][4]. However, some of these algorithms [4] are based on the reception from the multicast users of some sort of feedback information in order to take reactions at the sender side and perform adaptation of the transmitted flow. Other algorithms [3], instead, rely on layered video, where the video sequence is encoded over different channels: a base layer channel is in charge of transporting the video flow at its minimum quality and a set of enhancement layers channels carries progressive refinements of the video. With layered video at play, every user can independently join the optimum number of channels based on the constraints on its network segment, i.e., on its experienced bandwidth, round trip delay and error rate.

However, it is worth observing that all of these schemes operate at the video frame level, i.e., the knowledge of video frames content and boundaries is needed to efficiently exploit these algorithms. Unfortunately, over 3G networks, this knowledge is often impossible or difficult to be achieved. In such a system, in fact, the information regarding the layers above and including IP is often not available, e.g., at gateways and forwarding nodes [5], and for this reason error control schemes based on IP and/or video frame level information are difficult to be implemented. Beside that, the delays over such networks are considerable, due to e.g., channel coding and interleaving, and this makes the usage of feedback information ineffective.

Over such networks, instead, the only available information is about link layer data flows, where packets coming from higher layers (IP) are segmented in usually smaller sized packets (PDUs) and transmitted in sequential order and without any knowledge about their position within the video frame sequence. The link layer flows are assembled at the base station controller (BSC) and transmitted over the wireless channel by the base stations (BSs). The error control of these flows is performed both at the physical (L1) and link layer (L2) levels. At the physical level, channel coding and interleaving are used for this purpose, whereas at the link layer level (L2), erroneous and/or missing PDUs are often retransmitted 
thanks to ARQ/HARQ algorithms [5]. Hence, wireless errors are locally recovered thanks to $\mathrm{L} 1$ and $\mathrm{L} 2$ techniques. We think that in the single user single channel case this local recovery, due to the facts highlighted above, is an efficient and reasonable way to account for wireless channel impairments. In the multicast case, however, additional problems arise. First of all, the same information has to be conveyed to multiple users that are, in general, characterized by different and independent channel error patterns. Moreover, the residual errors after L1 processing are to be corrected to meet the desired QoS. The main additional problems in this case are

- To convey the common multicast flow to multiple users in a system-efficient and scalable way.

- To provide a simple and still effective way to perform a distributed error recovery of different errors patterns.

A widely accepted solution to cope with the first point above is to transmit the multicast flow over a downlink common channel $(\mathrm{CCH})$ [1]. In this way, all the interested users can receive the wanted information and only one spreading sequence is allocated for all of them. Multiple dedicated channels, in fact, would be system ineffective since a single channel would have to be allocated for every user to transmit the same information, thereby dramatically reducing the available system resources. However, this solution poses a further challenge in the error recovery phase, since different error patterns have to be corrected through a single forward channel. One possible solution to this problem, also adopted in [1], is to implement a multi dimensional HARQ scheme, where the single flow is transmitted over a common channel and some feedback information is collected from all the multicast users in the cell. Every users, before asking for a retransmission, tries to independently recover the error using a packet-based FEC scheme. At the base station controller, retransmission requests are collected and the decisions about the packets that have to be retransmitted are taken based on several different policies. One of such policies, for instance, could be to always retransmit a requested packet, even if that packet was requested by only one user. As shown in [1] this policy corresponds to a fully reliable scheme, but its channel efficiency is very low as the number of multicast users increases. Other strategies also account for the channel quality at the different users by trading retransmission fairness and channel efficiency (decreased by the retransmitted data).

However, the major drawback of these schemes is the need for L2 feedback channels. These channels are, in fact, expensive for the operator ${ }^{1}$ and, beside that, also give rise to side effects such as the NACK implosion [6]. The feedback also implies fairness problems. For instance, a "bad" user, i.e., a user that often asks for retransmissions could degrade the quality of all the remaining good users in the cell, since retransmitted packets are mixed into the same common channel, thereby diminishing the channel efficiency.

In this paper, motivated by what discussed above, we advocate to achieve error control through the usage of multiple

\footnotetext{
${ }^{1} \mathrm{~A}$ low rate dedicated uplink channel has to be allocated for each user to carry feedback information such as retransmission requests.
}

common channels. In particular, we exploit packet-based FEC techniques by opportunely spreading the redundancy over parallel channels and letting the users to independently choose the number of channels to be joined, depending on their experienced channel quality as well as their desired QoS level.

The paper is organized as follows. In the next section II, we present the algorithm for the delivery of multicast streaming flows. In section III, we introduce the system model that has been considered to analytically characterize the proposed scheme. In section IV, we introduce the analytical model and in section $\mathrm{V}$ we report and discuss some of the obtained results. Finally, section VI concludes the paper.

\section{Algorithm For Multicast Streaming through MULTI-CHANNEL TRANSMISSIONS}

Consider that $\mu_{1} \geq 1$ common channels are available for the transmission of the multicast streaming flow in a $3 \mathrm{G}$ cell. Moreover, consider that this flow is delivered to the BSC of a $3 \mathrm{G}$ cellular system in a timely and error-free manner. In fact, our main concern here is to devise an effective scheme for the error recovery over the wireless channel between the base station and the $3 \mathrm{G}$ mobile users. As said above, the flow is assembled at the BSC into a L2 PDU sequence. In our scheme, we propose to encode this PDU sequence using a packet-based FEC algorithm (RSE encoder), similar to the one in [2] and working as follows.

Suppose to have $K$ source information PDUs, $\left\{d_{1}, d_{2}, \ldots, d_{K}\right\}$. The RSE encoder takes these $K$ packets and produces $N-K$ parity packets $\left\{p_{1}, p_{2}, \ldots, p_{N-K}\right\}$. The $\mathrm{RSE}$ decoder, at the receiver side, can then reconstruct the data packets, $\left\{d_{1}, d_{2}, \ldots, d_{K}\right\}$, whenever it has received any $K$ out of the $N$ packets $\left\{d_{1}, \ldots, d_{K}, p_{1}, \ldots, p_{N-K}\right\}$. Most RSE implementations send the first $K$ original data as the first $K$ packets to simplify decoding (systematic code).

The algorithm we propose is very simple. We subdivide the PDU sequence in groups of $K$ packets that we pass to the RSE encoder in order to produce a total of $N$ PDUs. Further, according with the number of available common channels, $\mu_{1}$, we subdivide the $N-K$ redundancy PDUs in $\mu_{1}$ sets, that is $N-K=\sum_{n=1}^{\mu_{1}} H_{n}$, where $H_{n}$ is the number of redundancy PDUs assigned to the $i$-th channel. At this point, we send the first $K+H_{1}$ PDUs over the first channel, and the remaining $H_{n}$, $n=\left\{2, \ldots, \mu_{1}\right\}$, PDUs over every other channel. Moreover, to reduce the effect of error bursts, we introduce a delay of $\tau \geq 0$ slots between every pair of channels, where a single slot duration corresponds the constant PDU transmission time.

To sum up, the available redundancy is spread across different channels. This has the following advantages

- The good users, i.e., the users experiencing error-free channel periods do not need to join all of the $\mu_{1}$ channels. They can decide to join the first channel with null or limited decoding delay.

- Every user, according to its own channel error process can independently choose the number of common channels to be joined, that in turn, translates into the number of redundancy packets that the user will receive. 


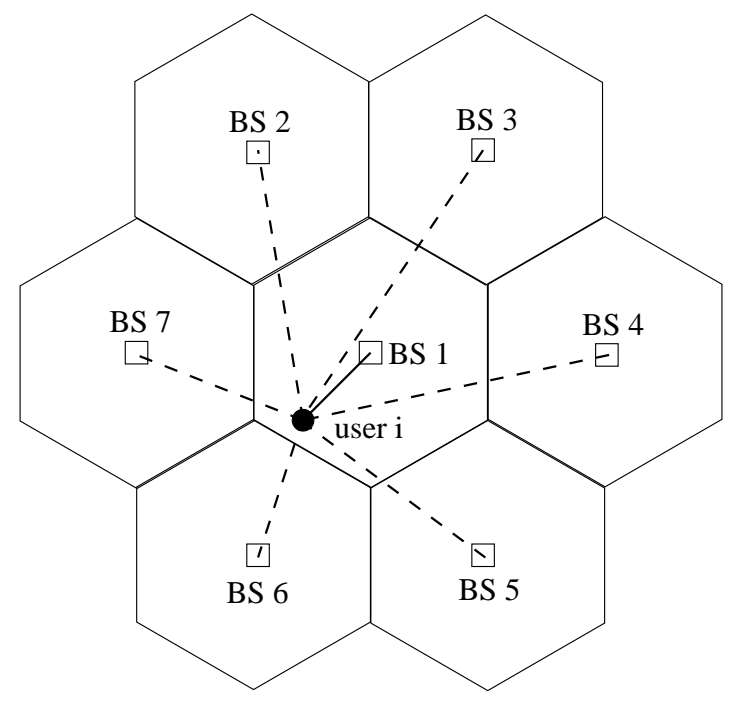

Fig. 1. Reference cellular network: user and BSs positioning.

- The decision of a user does not affect the quality and/or the channel efficiency of any other user in the system.

- While the bandwidth of the first channel is constrained by the bandwidth of the video stream under transmission, the bandwidth of the additional channels can be reduced, due to the lower number of packets transmitted over such channels. This also leads to a more robust transmission of the redundancy PDUs.

Of course, the key point is to correctly distribute the redundancy among the available channels. Some examples are given later on in section $\mathrm{V}$.

\section{SYSTEM MODEL}

In this section we present the interference and channel models considered in our analysis for the performance investigation of the transmission technique discussed above. Our aim here is to model the dynamic behavior of the single user channel, by jointly considering path loss, user interference and fast fading. The multi-user interference is modeled in section IIIA, where system load, base station positioning and path loss are accounted for. Furthermore, in section III-B we report the procedure that has been considered to track the channel fading propagation phenomena. This procedure considers the multiuser interference together with the model in [7] to characterize the faded channel through a Markov model.

\section{A. User Interference Model}

We assume to be operating in a W-CDMA multi-cellular environment, where several cells are distributed according to a hexagonal pattern, one base station is present at the center of every cell and a target downlink multicast flow has to be transmitted to a set of interested users. Users are considered to be randomly placed according to a planar Poisson process of intensity $\lambda$, i.e., the average number of users within an area $\mathcal{A}$ is given by $\lambda \mathcal{A}$, whereas the probability to have exactly $n$ nodes in this area is derived as $\left((\lambda \mathcal{A})^{n} / n !\right) \exp (-\lambda \mathcal{A})$.
We consider that a maximum downlink power $P_{\max }$ can be allocated in every cell, where each channel has a maximum transmission power equal to $P_{t x}$. According to $P_{\max }$, the number of maximum power channels that can be allocated in a single cell is given by $N_{c h}=P_{\max } / P_{t x}$. The tracked multicast flow consumes $\mu_{1} \geq 1$ common channels at the maximum transmission power $P_{t x}$. In fact, our choice here is to carry the multicast streams over common channels, where fast power control is usually not performed. In this case, to improve reliability, it is more convenient to keep the transmission power at a constant value. This power is usually considered to be equal to the maximum power level $P_{t x}$. Associated to $\mu_{1}$ there is a fraction $\varphi_{1}$ of the total downlink power, whereas a fraction $\varphi_{2}$ of $P_{\max }$ can be allocated to other transmissions and is regarded here as system interference. Observe that, $\varphi_{1}+\varphi_{2} \leq 1$. Our goal here, is to characterize the performance of the set of multicast users within a target cell. To this aim, we need to consider both the inter- $\left(I_{e x t}\right)$ and intra-cell $\left(I_{i n t}\right)$ interference, where the first contribution is estimated by considering the transmissions originated in the first circle of cells that are surrounding the target one (see Fig. 1). Besides that, the intracell interference has also to be taken into account. In fact, the theoretically orthogonal codes used for the transmission to the users within the target cell, at their reception, are no more orthogonal because of the multi-path propagation effects. This fact is accounted in our analysis by means of the orthogonality factor $\alpha \in[0,1]$.

The amount of interference is modulated through the parameter $\varphi_{2}$. The interference for user $i$ in the target cell and for a single multicast channel, namely $I_{t o t}(i)$, is therefore evaluated as [8]

$$
\begin{aligned}
I_{t o t}(i) & =I_{\text {int }}+I_{\text {ext }}= \\
& =\alpha\left[\left(\mu_{1}-1\right) P_{t x}+\varphi_{2} P_{\max }\right] L_{1 i}+ \\
& +\sum_{j=2}^{7} \varphi_{2} P_{\max } L_{j i}
\end{aligned}
$$

where the target cell is identified as cell 1 , cells numbered from 2 to 7 are the surrounding ones (Fig. 1), $L_{j i}$ is the propagation path loss between base station $j$ and user $i$ and $\alpha$ is the orthogonality factor introduced above. The path loss $L_{j i}$ is modeled through the well-known Hata model [9], where $L_{j i}=A d_{j i}^{-\beta}, A=0.001, \beta=3.5$ and $d_{j i}$ is the Euclidean distance between base station $j$ and user $i$. The signal to interference noise ratio $\left(S I R_{i}\right)$ for a single channel of user $i$ is then derived as follows

$$
S I R_{i}=\frac{E_{b}}{I_{o}}=\frac{W}{R} \cdot \frac{P_{t x} L_{1 i}}{I_{t o t}(i)+N_{o} W}
$$

where $W$ represents the chip rate of the CDMA signal, $R$ is the data rate allocated for the channel and $N_{o} W$ is the thermal noise power. $S F=W / R$ represents the user's spreading factor.

\section{B. Markov Channel Model}

We assume the time slotted, where the slot duration corresponds to the single packet (PDU) transmission time. The dynamic behavior of the single multicast channel packet error 


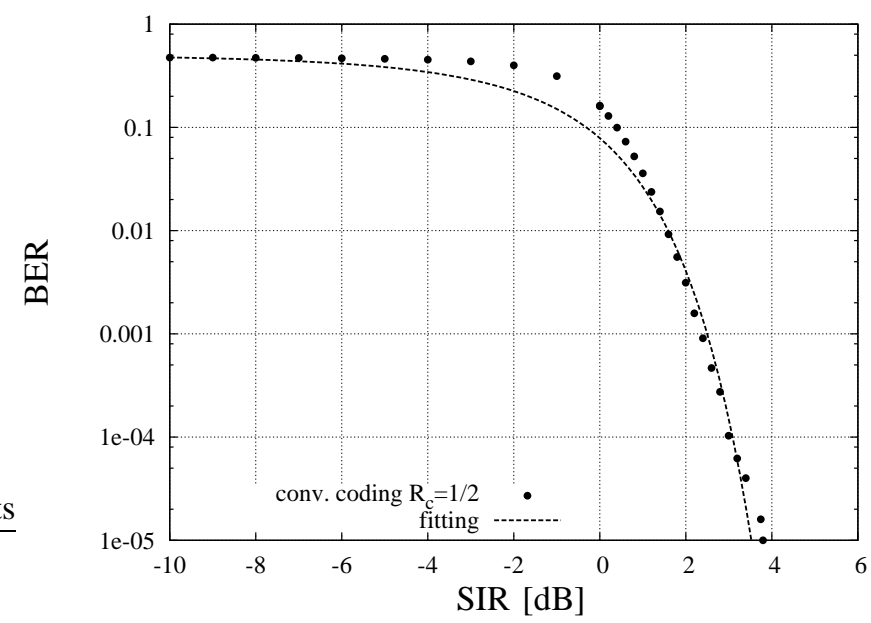

Fig. 2. Bit error rate after coding for $R_{c}=1 / 2$ : simulated points and fi tting.

process at user $i$ is modeled by means of a Gilbert Elliot channel [10], where state 0 and state 1 are the good and bad states, respectively. Further, we consider $P_{e 0}$ and $P_{e 1}$ to be the PDU error probabilities in state 0 and state 1 , respectively. The embedded Markov chain $\mathbf{P}$ of the channel error process is obtained as in [7]

$$
\mathbf{P}=\left(\begin{array}{cc}
p_{00} & p_{01} \\
p_{10} & p_{11}
\end{array}\right)
$$

Let $\gamma$ be a threshold characterizing the physical transmission and the coding technique. In practice, for a given channel situation, the $i$-th symbol is assumed to be correctly received whether the fading envelope is above $\gamma$, where $F=1 / \gamma$ is referred to as fading margin [7]. Let $S I R_{t h}$ be the $S I R$ that suffices to correctly decode a symbol. In our scenario, once $S I R_{t h}$ has been fixed, $\gamma$ depends on the useful received power $P_{t x} L_{1 i}$, on the system interference and on thermal noise at the receiver. $\gamma$ for user $i$ is therefore derived as

$$
\gamma=\frac{S I R_{t h}}{S F} \cdot \frac{I_{t o t}(i)+N_{o} W}{P_{t x} L_{1 i}}
$$

In practice, with the previous Eq. (4) we get the maximum value of the fading envelope needed to fulfill the QoS requirement dictated by $S I R_{t h}$. For what concerns the packet error probabilities in the good and bad states of the Gilbert Elliot channel, we account for a physical layer convolutional coding with code rate $R_{c}$. In the good state (state 0 ), we assume a perfect error recovery and therefore we assign $P_{e 0}=0$. On the other hand, in the bad state we compute the related bit error rate $P_{b 1}$ by means of a pre-computed curve that relates the actual $S I R$ at user $i\left(S I R_{i}\right.$, see Eq. (2)) to the bit error rate after coding. An example of such a curve and of its possible fitting is given in Fig. 2, where we considered an half rate $\left(R_{c}=1 / 2\right)$ convolutional code as specified in [11]. In this case, the error probability curve can be accurately fitted by a modified erfc $(\cdot)$ function as follows

$$
P_{b 1}(S I R)=\frac{1}{2} \operatorname{erfc}\left(\sqrt{S I R^{\zeta}}\right)
$$

where $\zeta=2.72$ and the fitting as been performed by giving more importance to the tail of the error rate curve. The packet error rate in state 1 is finally derived as $P_{e 1}=1-\left(1-P_{b 1}\right)^{M}$, where $M$ is the length in bit of a single packet.

\section{Analytical Framework}

\section{A. First Channel}

In this section we derive an analytical expression to evaluate the successful decoding rate of the FEC blocks transmitted over the first common channel. We consider that the time is slotted, where the slot duration corresponds to the single PDU transmission time. Moreover, we consider that FEC blocks consisting of $K+H_{1}$ PDUs are transmitted back-to-back over such channel, where $K$ and $H_{1}$ represent the original data packets and the added redundancy, respectively.

Let $\psi_{i j}(k, n, h), i, j \in\{0,1\}$ be the probability, for user $h$, that $k$ slots in $\{1,2, \ldots, n\}$ are in state 0 and the channel state is $j$ at time $n$, given that the channel state was $i$ at time 0 . This is a well-known function that can be derived either in recursive [12] or close [13] form. Observe that the function $\psi(\cdot)$ depends on the selected user $h$ through its position and Doppler frequency $f_{d}^{h}$. However, in order to proceed with our analysis we need to derive the following function $\phi_{i j}(k, n, h)$ that corresponds to the probability, for user $h$, that $k$ slots in $\{1,2, \ldots, n\}$ are successful and the channel state is $j$ at time $n$, given that the channel state was $i$ at time $0 . \phi(\cdot)$ is readily derived from $\psi(\cdot)$ and the packet error probabilities in state 0 $\left(P_{e 0}\right)$ and $1\left(P_{e 1}\right)$

$$
\phi_{i j}(k, n, h)=\sum_{\xi=0}^{n} \psi_{i j}(\xi, n, h) f[n-k, n-\xi, n]
$$

where $\xi$ and $e=n-k$ are used to track the number of slots in state 0 and the number of erroneous slots in $\{1,2, \ldots, n\}$, respectively. $f[n-k, n-\xi, n]$ is the probability of having $n-k$ erroneous slots out of $n$ given that $b=n-\xi$ slots are in state 1. The function $f[\cdot]$ is derived as

$$
f[e, b, n]=\sum_{u=\max (e-n+b, 0)}^{\min (e, b)} \mathcal{E}[1, u \mid b] \mathcal{E}[0, e-u \mid n-b]
$$

where $\mathcal{E}[i, r \mid s]=\left(\begin{array}{l}s \\ r\end{array}\right) P_{e i}^{r}\left(1-P_{e i}\right)^{s-r}$. The correct decoding of a FEC block occurs when at least $K$ PDUs are correctly received out of the $K+H_{1}$ transmitted. The probability associated with this event is the same of having at least $K$ successful slots over $K+H_{1}$, averaged over all the possible evolutions of the channel process during these $K+H_{1}$ slots. The decoding success probability for user $h$ is found as

$$
P_{1}^{\mathrm{ok}}\left(f_{d}^{h}, \theta, r\right)=\sum_{i_{1}=0}^{1} \pi_{i_{1}} \sum_{j_{1}=0}^{1} \sum_{\xi_{1}=K}^{K+H_{1}} \phi_{i_{1} j_{1}}\left(\xi_{1}, K+H_{1}, h\right)
$$

where $f_{d}^{h}, \theta$ and $r$ are the Doppler frequency and the polar coordinates of user $h$ in the target cell. $\pi_{i_{1}}$ is the channel steady state probability associated with state $i_{1} \in\{0,1\}$. 


\section{B. n-th Channel}

Observe that every further channel we introduce to improve the quality of the multicast flow is transmitted by a codedivision access technique, by sharing the "time" and "frequency" resources with the first considered channel. For this reason, these channels are expected to be affected by an error process that is very correlated with respect to the one striking on the first channel. The delay shifts of $\tau$ slots that we introduce between every channel pair have the role of alleviating such a correlation.

Let us observe that the probability of a correct decoding at the $n$-th channel is, just as in the first channel case

$$
P_{n}^{\mathrm{ok}}\left(f_{d}^{h}, \theta, r\right)=\sum_{i_{1}=0}^{1} \pi_{i_{1}} \sum_{j_{n}=0}^{1} \sum_{\xi_{n}=K}^{K+\sum_{u=1}^{n} H_{u}} P\left[\xi_{n}, i_{1}, j_{n}\right]
$$

where $i_{1}$ and $j_{n}$ represent the channel states of the slot preceding the first transmission over the first channel (slot 0), and the slot occupied by the last of the $H_{n}$ packets transmitted over the $n$-th channel (slot $n$ ), respectively. $P\left[\xi_{n}, i_{1}, j_{n}\right]$ is the probability that a total of $\xi_{n}$ PDUs are correctly received out of the $K+\sum_{u=1}^{n} H_{n}$ transmitted over the first $n$ ones and that the channel slot $n$ is in state $j_{n}$ given that the channel slot 0 is in state $i_{1} . P\left[\xi_{n}, i_{1}, j_{n}\right]$ can be recursively evaluated as follows

$$
\begin{aligned}
P\left[\xi_{n}, i_{1}, j_{n}\right] & =\sum_{j_{n-1}, i_{n}}^{1} \sum_{\xi_{n-1}=0}^{Z}\left[P\left[\xi_{n-1}, i_{1}, j_{n-1}\right] \times\right. \\
& \left.\times p_{j_{n-1} i_{n}}(\tau) \phi_{i_{n} j_{n}}\left(\xi_{n}-\xi_{n-1}, H_{n}, h\right)\right] \\
P\left[\xi_{1}, i_{1}, j_{1}\right] & =\phi_{i_{1} j_{1}}\left(\xi_{1}, K+H_{1}, h\right)
\end{aligned}
$$

where $\xi_{n} \in\left\{0,1, \ldots, K+\sum_{u=1}^{n} H_{u}\right\}, Z=\min \left(\xi_{n}, K+\right.$ $\left.\sum_{u=1}^{n-1} H_{u}\right), p_{j_{n-1} i_{n}}(\tau)$ is the $\left(j_{n-1}, i_{n}\right)$-th entry of the $\tau$ step channel transition matrix $\mathbf{P}^{\tau}$ and $H_{n}$ is the number of redundancy packets transmitted over the $n$-th channel.

\section{RESULTS}

The cellular environment considered to carry out the performance evaluation is the one presented in section III, where users are randomly placed according to a planar Poisson model. The PDU error process at every user $h$ is characterized by a two state Markov channel whose parameters are derived from the users' positions, mobility, i.e., Doppler frequency $f_{d}^{h}$ and degree of interference affecting the system $\varphi_{2}$.

The system performance is evaluated through the mean users' code failure probability that is derived as follows

$$
\mathcal{F}=\frac{1}{N_{f_{d}}} \sum_{h=1}^{N_{f_{d}}} \int_{0}^{2 \pi} \int_{0}^{R_{\text {cell }}}\left\{\frac{1-P_{n}^{\mathrm{ok}}\left(f_{d}^{h}, \theta, r\right)}{\pi R_{\text {cell }}^{2}}\right\} d \theta d r
$$

where $R_{\text {cell }}$ is the cell radius, $N_{f_{d}}$ is the number of mobility classes considered in the analysis, where mobility classes are assigned by means of an uniform distribution. In particular, $f_{d}^{h}$ is randomly picked from the $N_{f_{d}}=10$ uniformly distributed values between 6 and $80 \mathrm{~Hz}$. In the equation above, we take the double expectation of the decoding failure probability over

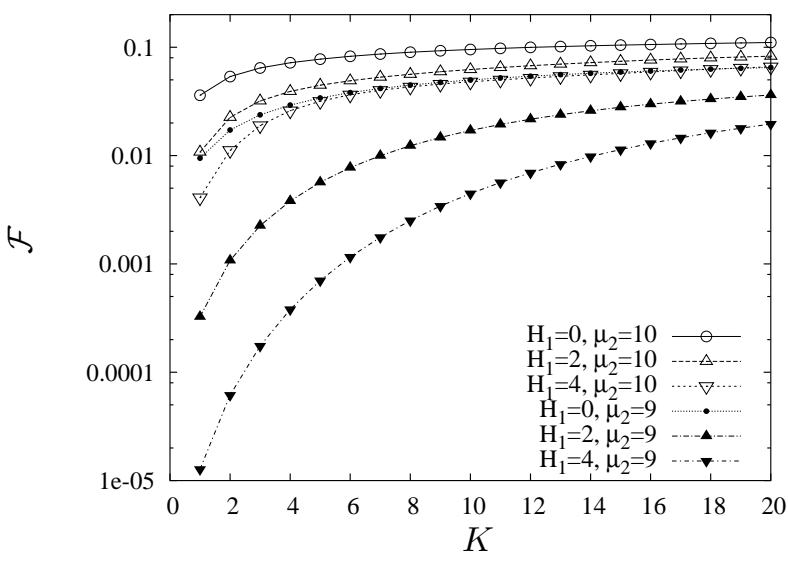

Fig. 3. $\mathcal{F}$ as a function of $K$ by varying $H_{1}$ and $\mu_{2}$.

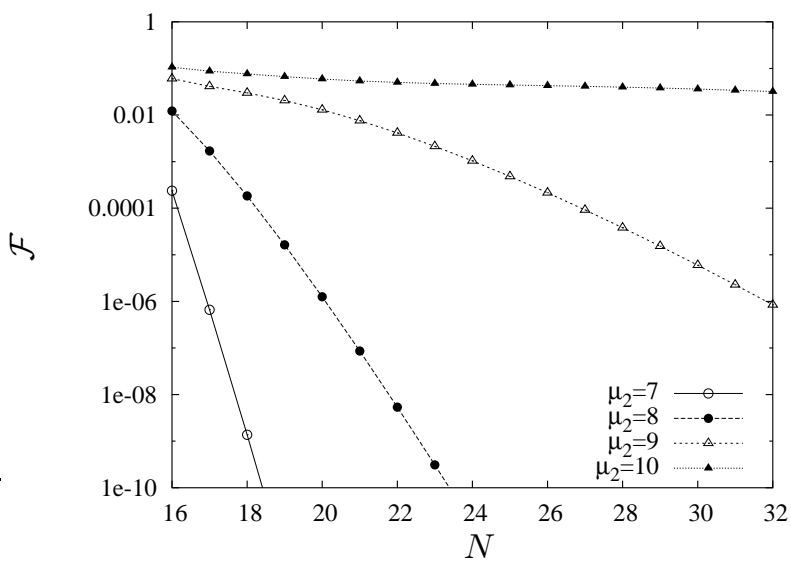

Fig. 4. $\mathcal{F}$ as a function of $N$ by varying $\mu_{2} . N=K=16$ corresponds to an uncoded channel, i.e., $H_{1}=0$.

user's positions and mobility classes. In the results presented next we considered $S F=32, P_{t x}=-5 \mathrm{dBm}, W=4.6848$ $\mathrm{MHz}$ [5], $R_{\text {cell }}=200 \mathrm{~m}, P_{\max }=11 P_{t x}, M=360$ bits, $\tau=10, \alpha=0.1$.

In Fig. 3, we plot $\mathcal{F}$ as a function of $K$ by considering a single channel and by varying its redundancy $H_{1}$. Moreover, we refer here to the number of interference channels in every cell as $\mu_{2}$, i.e., $\varphi_{2}=\left(\mu_{2} P_{t x}\right) / P_{\max }$ and we consider a single $\mathrm{CCH}$ to convey the multicast traffic, i.e., $\mu_{1}=1$. As expected $(S F=32)$, adding a single interfering channel ( $\mu_{2}=9 \rightarrow \mu_{2}=10$ ) causes a severe degradation of the users' performance. Moreover the utility of incrementing $H_{1}$, in terms of $\mathcal{F}$, decreases as $\mu_{2}$ increases. It is also worth noting that, reasonable values $(K / N \geq 0.8)$ of $\left(K, H_{1}\right)$, e.g., $K=16$ and $H_{1}=4$, give a benefit of about one order of magnitude with respect to $H_{1}=0$ (no packet level coding).

In Fig. 4, we report $\mathcal{F}$ as a function of $N=K+H_{1}$ by considering $K=16$. As above, we can observe the gain derived from an increased $H_{1}$ and the large impact of $\mu_{2}$. At the maximum interference level $\left(\mu_{2}=10\right.$, i.e., $P_{t x}^{\text {tot }}=$ $\left.\left(\mu_{1}+\mu_{2}\right) P_{t x}=11 P_{t x}=P_{\max }\right)$, the added redundancy is useless and the error rate is too high to support the transmission 


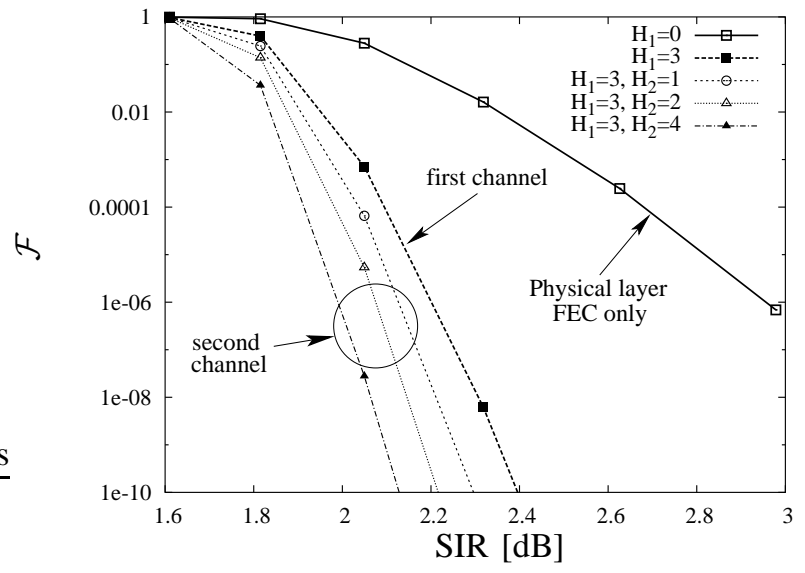

Fig. 5. $\mathcal{F}$ as a function of the user's SIR.

of multimedia flows. However, observe how such a redundancy is, instead, very effective as $\mu_{2} \leq 8$.

As a last graph, in Fig. 5 we report some results concerning the impact of a second channel. In particular, we consider a single multicast flow to be transmitted over two $\mathrm{CCH}$ channels. On the first channel, both data $(K)$ and redundancy $\left(H_{1}\right)$ are transmitted to provide a first level of protection, whereas a controlled amount of redundancy $\mathrm{H}_{2}$ is transmitted over the second $\mathrm{CCH}$. This second level of protection can be independently exploited by all users in the cell, if needed. The performance in Fig. 5 is reported as a function of the average $S I R$ perceived by the user, therefore in such a case, $\mathcal{F}$ is averaged over the mobility classes only. In fact, in our network model, there is a correspondence between user position and average $S I R$. In general, the packet level coding provides substantial performance improvements with respect to considering a physical layer FEC only. However, as $S I R \leq 1.8$ $\mathrm{dB}$ the physical layer coding fails, thereby giving raise to a too high packet error rate which, in turn, makes packet level coding ineffective. Note also that, as $S I R \geq 1.8$, a second $\mathrm{CCH}$ channel with a limited amount of redundancy $\left(H_{2}=4\right)$ can reduce the decoding failure probability of about two order of magnitude. It is therefore feasible to improve performance by means of a low rate secondary $\mathrm{CCH}$ channel.

The following last observation is in order here. The bit error probability needed to achieve a good visual quality of video streams (e.g., H26L video coding) is usually lower than $10^{-6}$ [14] that, in turn, translates into a maximum packet error rate after coding of about $3.5 \times 10^{-4}(M=360)$. Now, considering the worst case where if a FEC block of $N$ PDUs is undecodable (with probability $\mathcal{F}$ ) then all the $K$ data packets therein are undecodable as well $^{2}$, we can approximate the packet error probability after coding with $\mathcal{F}$. Hence, the video flow in such a case is played with a good quality as $\mathcal{F} \leq 3.5 \times 10^{-4}$.

From this preliminary study we can therefore conclude that

\footnotetext{
${ }^{2}$ Note that using a systematic code and appending a CRC check fi eld to every packet, the correctly received data packets can be recognized as correct and passed to the application level.
}

the usage of packet level coding together with its spreading over multiple channels is feasible and is also a promising technique to improve the quality of multimedia flows.

\section{CONCLUSiOnS}

In this paper, we proposed and discussed an error recovery technique for the efficient delivery of multicast streaming flows in third generation cellular networks. With respect to previous approaches [1], we do not allow for the retransmission of lost packets to improve performance. Instead, we propose the usage of packet level FEC [2] over multiple parallel common channels. This coding strategy is exploited in conjunction with physical layer coding to further reduce error rates. Such technique is then investigated by analytical results, by proposing a system model able to capture the dynamic behavior of users' channel error processes and by defining a suitable metric to weight the system performance.

The advantage of our scheme is that error recovery is performed independently at each user that, based on its own experienced quality, can decide the right number of channels to be joined. This procedure does not need any feedback channel with obvious advantages in terms of delay, system complexity and costs. For these reasons, such technique is appealing for being actually implemented in third generation cellular systems.

A further investigation on the impact of the time shift $\tau$ between channels and of the effectiveness of the technique over accurate channel traces is left for future research.

\section{REFERENCES}

[1] M. Rossi, M. Zorzi, and F. H. P. Fitzek, 'Investigation of Link Layer Algorithms and Play-Out Buffer Requirements for Effi cient Multicast Services in 3G Cellular Systems," Accepted for Publication in IEEE PIMRC 2004, Barcelona, Spain, Sept. 2004.

[2] L. Rizzo, 'Effective Erasure Codes for Reliable Computer Communications Protocols," ACM Computer Communication Review, vol. 27, no. 2, pp. 24-36, Apr. 1997.

[3] W.-T. Tan and A. Zakhor, "Video multicast using layered FEC and scalable compression," IEEE Trans. Circuits Syst. Video Technol., vol. 11, pp. 373-386, Mar. 2001.

[4] J. Bolot, T. Turletti, and I. Wakeman, "Scalable Feedback Control for Multicast Video Distribution in the Internet," Computer Comm. Review, vol. 24, no. 4, pp. 58-67, Oct. 1994.

[5] H. Holma and A. Toskala, WCDMA for UMTS: Radio Access for Third Generation Mobile Communications. John Wiley \& Sons, Ldt, 2000.

[6] D. D. Lucia and K. Obraczka, "Multicast Feedback Suppression using Representatives," in Proceedings of IEEE INFOCOM, Kobe, Japan, Sept. 1997, pp. 463-470.

[7] M. Zorzi, R. R. Rao, and L. B. Milstein, "On the accuracy of a fi rst order Markov model for data transmission on fading channels," in Proceedings of IEEE ICUPC, Tokyo, Japan, Nov. 1995, pp. 211-215.

[8] A. de Hoz and C. Cordier, "W-CDMA Downlink Performance Analysis," in Proceedings of IEEE VTC 1999-Fall, vol. 2, Amsterdam, The Netherlands, Sept. 1999, pp. 968-972.

[9] J. G. Proakis, Digital Communications, 2nd ed. New York: McGrawHill, 1999.

[10] M. Mushkin and I. Bar-David, "Capacity and Coding for the GilbertElliot Channels," IEEE Trans. Inform. Theory, vol. 35, no. 6, pp. 12771290, Nov. 1989.

[11] '3GPP Third Generation Partnership Project," [Available] http: / / www. 3gpp.org.

[12] R. A. Howard, Dynamic Probabilistic Systems. New York: Wiley, 1971.

[13] M. Zorzi and R. Rao, 'Lateness probability of a retransmission scheme for error control on a two-state Markov channel," IEEE Trans. Commun., vol. 47, pp. 1537-1548, Oct. 1999.

[14] F. Fitzek, M. Rossi, and M. Zorzi, 'Error Control Techniques for Effi cient Multicast Streaming in UMTS Networks," in Proceedings of SCI 2003, Orlando, Florida, US, July 2003. 